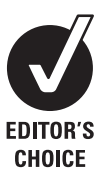

CHOICE

\title{
Allogenic mesenchymal stem cells transplantation in refractory systemic lupus erythematosus: a pilot clinical study
}

\author{
Jun Liang, ${ }^{1}$ Huayong Zhang, ${ }^{1}$ Bingzhu Hua, ${ }^{1}$ Hong Wang, ${ }^{1}$ Liwei Lu, ${ }^{2}$ Songtao Shi, ${ }^{3}$ \\ Yayi Hou, ${ }^{4}$ Xiaofeng Zeng, ${ }^{5}$ Gary S Gilkeson, ${ }^{1,6}$ Lingyun Sun ${ }^{1}$
}

\begin{abstract}
- Additional data are published online only. To view these files please visit the journal online (http://ard.bmj.com)

'Department of Rheumatology and Immunology, The Affiliated Drum Tower Hospital of Nanjing University Medical School, Nanjing, China

2Department of Pathology, The University of Hong Kong, Hong Kong, China

${ }^{3}$ Center for Craniofacial

Molecular Biology, University

of Southern California School

of Dentistry, Los Angeles,

California, USA

${ }^{4}$ Immunology Laboratory,

Nanjing University Medical

School, Nanjing, China

${ }^{5}$ Department of Rheumatology, Peking Union Medical College,

Chinese Academy of Medical

Sciences, Beiijing, China

${ }^{6}$ Division of Rheumatology,

Medical University of South

Carolina, Charleston, South

Carolina, USA
\end{abstract}

\section{Correspondence to}

Dr Lingyun Sun, Department of

Rheumatology and Immunology,

The Affiliated Drum Tower

Hospital of Nanjing University

Medical School, 321 Zhongshan

Road, Nanjing, Jiangsu 210008,

China;

lingyunsun2001@yahoo.com.cn

Dr Gary S Gilkeson, Department of Rheumatology, 96 Jonathan Lucas Street, Suite 912 Medical University of South Carolina, Charleston, South Carolina

29425, USA;

gilkeson@musc.edu

$\mathrm{JL}$ and $\mathrm{HZ}$ authors contributed equally to this work.

Accepted 17 May 2010

\section{ABSTRACT}

Objective To determine the safety and efficacy of allogeneic mesenchymal stem cell transplantation (MSCT) in refractory systemic lupus erythematosus (SLE). Methods A total of 15 patients with persistently active SLE underwent MSCT. Outcome was evaluated by changes in the SLE disease activity index (SLEDAI), serological features (anti-nuclear antibodies and antidouble-stranded DNA (anti-dsDNA)), renal function and percentage of peripheral blood regulatory T cells.

Results From 11 March 2007 to 4 November 2008, 15 patients with persistently active SLE were enrolled and underwent MSCT. The mean follow-up period was $17.2 \pm 9.5$ months. A total of 13 patients have been followed for more than 12 months. All patients clinically improved following treatment with mesenchymal stem cells with a marked decrease in the SLEDAI score and $24 \mathrm{~h}$ proteinuria. At 12-month follow-up, SLEDAl scores decreased from $12.2 \pm 3.3$ to $3.2 \pm 2.8$ and proteinuria decreased from $2505.0 \pm 1323.9$ to $858.0 \pm 800.7$ $\mathrm{mg} / 24 \mathrm{~h}$ (all $p<0.05$, by paired t test, $\mathrm{n}=12$ ). At 1 -year follow-up in 13 patients, 2 had a relapse of proteinuria, while the other 11 continue to have decreased disease activity on minimal treatment. Anti-dsDNA levels decreased. Improvement in glomerular filtration rate was noted in two patients in which formal testing was performed. Non-renal-related manifestations also improved significantly. No serious adverse events were reported.

Conclusion Allogeneic MSCT in patients with refractory lupus resulted in amelioration of disease activity, improvement in serological markers and stabilisation of renal function. MSCT appears beneficial in treatment of patients with SLE refractory to conventional treatment options.

\section{INTRODUCTION}

Systemic lupus erythematosus (SLE) is an autoimmune inflammatory disease with multiorgan involvement including the kidney, brain, lung and haematopoietic systems. Lupus nephritis (LN) is a common major organ manifestation and is a significant cause of morbidity and mortality. The most widely and classically used immunosuppressive therapies, notably corticosteroids and cyclophosphamide (CYC), have led to a significant improvement in survival over the last few decades and decreased the progression to end-stage multiorgan failure. Both agents, however, are associated with significant side effects including increased susceptibility to infection. Other immunosuppressants, such as mycophenolate mofetil (MMF) and rituximab, appear to be effective in resistant SLE, but the cost is high and they also have significant side effects. ${ }^{1-4}$ Even with use of these potent immunosuppressive regimens, some patients are refractory with progressive organ-damaging and life-threatening disease, including LN. Currently, haematopoietic stem cell (HSC) transplantation is used in some cases of refractory SLE, which results in disease improvement in most cases, ${ }^{5}$ but also causes significant morbidity and mortality. The most common complications, including mucositis, transplantation-related infection and lung injury, have led to concerns regarding widespread use of this procedure in lupus. Given these current treatment limitations, new therapies are needed with enhanced efficacy and less toxicity than current treatment standards.

Mesenchymal stem cells (MSCs) are multipotential non-haematopoietic progenitor cells capable of differentiating into multiple cell lineages including osteoblasts, chondrocytes, myoblasts, adipocytes, endothelial cells, neuron-like cells, cardiomyocytes and hepatocytes. ${ }^{7-11}$ Because of their low immunogenicity due to their lacking expression of costimulatory molecules, they are able to escape alloantigen recognition. In addition, MSCs can inhibit immune responses in vitro and in vivo in a dose-dependent, non-human leucocyte antigen (HLA)-restricted manner. ${ }^{12} 13$ Their immune modulatory effect is related to effects on dendritic cell, T cell and natural killer cell development and activation. ${ }^{14-16}$ In human cell cultures, the magnitude of suppression is not reduced when the MSCs are separated from the lymphocytes in transwell plates, indicating that cell-cell contact is not required. These properties make MSCs promising candidate cells for preventing rejection in organ transplantation and treatment of autoimmune disease.

Over 10 years ago, Ikehara clarified the differences between normal and abnormal HSCs concluding that autoimmune diseases are 'stem cell disorders'. ${ }^{17}$ Recently, we showed functional abnormalities exist in bone marrow-derived MSCs (BM-MSCs) from patients with SLE and MRL/ lpr mice. ${ }^{18} 19$ MSCs from patients with SLE grew more slowly than those of normal controls, aged more quickly and lost vitality sooner during passage. Moreover, MSCs from patients with lupus compared to controls were defective in secreting cytokines (ie, transforming growth factor $\beta$ ) accompanied by downregulation of interleukin 6 (IL-6) and IL-7 mRNA expression. A recent article also confirmed defective phenotype, growth and 
immunomodulatory effects of MSCs from patients with SLE compared with those from age-matched and sex-matched healthy donors. ${ }^{20}$

We therefore hypothesised that SLE is potentially a MSCmediated disease. If this hypothesis is true, then allogenic rather than autologous BM-MSCs transplantation should be an effective treatment for patients with lupus. The study of Carrion et a ${ }^{21}$ showed that autologous MSCs treatment did not improve disease activity in two patients with SLE, consistent with our hypothesis. Many preclinical assessments were performed to investigate potential therapeutic effects and possible mechanisms of human bone marrow MSC transplantation (MSCT) on ameliorating autoimmune progression in MRL/lpr mice. ${ }^{19} 22$ Human MSC from healthy donors reduced the proliferation of T lymphocytes in vitro from MRL/lpr mice in a dose-dependent fashion. At 2 weeks after transplantation with allogenic MSCs, we detected significantly reduced serum levels of anti-doublestranded DNA (anti-dsDNA) antibodies and $24 \mathrm{~h}$ proteinuria in MRL/lpr mice compared with control groups. Moreover, flow cytometric analysis showed that MSCT decreased the total number of CD4 T cells while increasing the T helper (Th) 1 cell subpopulation when compared with controls. Histopathological examination showed significantly decreased renal pathology in MSC-treated mice. Immunohistochemical studies further revealed less expression of C3 in renal tissue after MSCT. Observations in animal models do not necessarily translate to humans. Therefore, this study was undertaken to determine whether MSCT can modulate the human immune system resulting in clinical improvement in patients with SLE refractory to current standards of treatment. Herein, we report short-term outcomes of 15 standard treatment refractory patients with SLE receiving MSCT in an unblinded clinical trial.

\section{METHODS}

\section{Patient eligibility}

From 11 March 2007 to 4 November 2008, 15 patients (14 women, 1 man) with SLE refractory to standard therapies were enrolled in an MSCT trial, which was approved by the Ethics Committee at Drum Tower Hospital and registered at http://ClinicalTrials.gov (identifier: NCT00698191). Informed consent was obtained from each patient and donor. All enrolled patients had at least 4 of 11 American College of Rheumatology criteria for SLE. Patient eligibility criteria also included one of the following features: (1) progressive and active disease with a Safety of Estrogens in Lupus Erythematosus: National Assessment (SELENA) SLE disease activity index (SLEDAI) score $\geq 8$, despite continuous treatment with intravenous pulse CYC with a total dosage of 400-800 mg every month for at least 6 months or oral MMF 1000-2000 mg/day for at least 3 months and continued daily dosage of more than $20 \mathrm{mg}$ of prednisone or its equivalent; (2) refractory immune-mediated thrombocytopoenia; (3) refractory LN. Refractory LN was defined either as proteinuria $\geq 1000 \mathrm{mg} / 24 \mathrm{~h}$, serum creatinine $(\mathrm{Cr}) \geq 1.5 \mathrm{mg} / \mathrm{dl}$ or decreased $\mathrm{Cr}$ clearance without end-stage renal failure in patients with WHO class IV/V glomerulonephritis despite 6 months of CYC or MMF.

\section{MSC culturing and transplanting}

The source of MSCs was BM-MSCs. Healthy donors between the ages of 18 and 40 years, who had no history of any significant illness and had no physical or mental disability, were selected from members of the patient's families without HLA matching, and all gave written informed consent. BM-MSCs were isolated by density gradient centrifugation and adherence methods as described previously with slight modification. ${ }^{16}$ Briefly, mononuclear cells were collected by gradient centrifugation and seeded at a density of $1 \times 10^{6}$ cells $/ \mathrm{cm}^{2}$ in growth medium containing Dulbecco's modified Eagle medium, low glucose (DMEM-LG; Gibco) and 10\% fetal bovine serum (FBS) (HyClone) (BM-GM). After 3 days of culture, non-adherent cells were removed and the medium was changed twice weekly thereafter. Once $60 \%$ to $80 \%$ confluence was reached, adherent cells were replated at a density of $10^{4}$ cells $/ \mathrm{cm}^{2}$ in BM-GM for expansion. After two passages, the cells were harvested. Flow cytometric analysis confirmed the cells expressed CD106, CD105, CD90, CD71, CD44, CD29, but not CD34, CD14, CD3 or CD45 (see supplementary material). The capacity of MSCs to differentiate along adipogenic and osteogenic lineages was evaluated as described previously. ${ }^{7}$ Rigorous purification and quality control were performed to ensure MSCs purity. In most experiments, analyses indicated $>95 \%$ purity (see supplementary material). Stem cells were used between passages 3 and 5 . All the patients received one intravenous infusion of $1 \times 10^{6}$ cells $/ \mathrm{kg}$ of body.

\section{Maintenance treatment}

All patients continued treatment with steroids at the time of infusion, with a taper of 5-10 mg every 2 weeks. Maintenance treatment 1 month after the MSCT included prednisone at 5-10 mg/day and CYC 0.4-0.6 g per 2-3 months (see supplementary material). As per the protocol, no other immunosuppressive medications were used to avoid treatment confounders affecting the outcomes of MSCT, unless disease relapsed. If the patient's status continued to improve, a withdrawal schedule was followed to taper off prednisone. All modifications of treatment were with the agreement of the treating rheumatologist.

\section{Assessment of disease status}

After MSCT, each patient returned for follow-up at 1 week, 1, 3, 6,12 and 18 months and then once every half a year thereafter. Evaluations performed at these times included a physical examination, serological testing, urinary protein excretion, measures of regulatory $\mathrm{T}$ cells (Tregs) and assessment of glomerular filtration rate (GFR) (if results were previously abnormal). A SLEDAI score was assigned at each baseline and follow-up visit for each patient. If a patient was not able to return for follow-up, medical records and laboratory measures were collected from their local doctor or medical facility.

\section{Laboratory tests}

Serum levels of anti-nuclear antibodies (ANAs) and anti-dsDNA antibodies were determined by indirect immunofluorescence using kits purchased from Euroimmun (Lübeck, Germany). The percentage of peripheral blood Tregs was analysed by flow cytometry (Becton Dickinson, San Jose, California, USA). Monoclonal antibodies directed against CD4 and Foxp3 were purchased from eBioscience. Flow data were analysed with CellQuest software (Becton Dickinson). GFR was determined by the method of Tc-99m diethylenetriamine penta-acetic acid (DTPA) renography.

\section{Statistical analysis}

The paired or unpaired t test was used for statistical comparison of before and after MSCT variables (SLEDAI, ANA, anti-dsDNA, 24 h proteinuria and Tregs) by GraphPad Prism 4 software (V.4.03; GraphPad, La Jolla, California, USA). A level of $p<0.05$ was considered statistically significant. 


\section{RESULTS}

\section{Patient profiles}

A total of 15 patients (14 women, 1 man) underwent allogeneic MSCT for refractory SLE. Their average age was 28.3 years (range 12-44). All had fulfilled eligibility criteria for MSCT. The average disease duration was 91.1 months. All patients had proteinuria. Table 1 displays patient demographics and drug regimens received at the time of MSCT. Table 2 shows clinical symptoms of the 15 patients at the time of MSCT.

\section{Assessment of disease activity}

The mean follow-up period was 17.2 \pm 9.5 months (range 3-36). In all, 13 patients were followed up for more than 12 months, including 1 for 36 months, 1 for 30 months, 4 for 24 months, 2 for 15 months and 5 for 12 months. Scores on the SLEDAI, a validated instrument of disease activity, improved significantly at 12 -month follow-up $(12.1 \pm 3.3$ vs $3.2 \pm 2.8$ at baseline, $\mathrm{n}=12, \mathrm{p}<0.05$ ) (figure 1 ). The SLEDAI score remained $<8$ in 12 of 13 patients at 12 -month follow-up. Four patients (cases 1, 5, 8 and 10) showed remarkable improvement with SLEDAI scores of 0 at 12 -month follow-up. Sustained clinical remission has lasted for another 24 months in one of these four patients (case 1). Two patients (cases 2 and 12) had a flare of disease with SLEDAI scores of 8 at their 24-month and 6-month follow-up, respectively (detailed data are shown in supplementary material). Of 15 patients, 1 (case 10) had discontinued all the other immunosuppressants with a gradual taper of prednisone dosage after MSCT. She had SLEDAI score of 2 with a regimen of prednisone $10 \mathrm{mg} /$ day at 3 -month follow-up and had SLEDAI score of 0 with prednisone $5 \mathrm{mg} /$ day at 12-month follow-up.

\section{Serological findings}

ANA and anti-dsDNA antibodies decreased after MSCT. Lower serum titres of anti-dsDNA (11 of 15 patients) became significant from baseline at 1 month $(p<0.05)$ and 3 months $(p<0.05)$ post transplant (figure 2). ANA antibodies also improved in parallel with anti-dsDNA, with the titre falling in nine patients at 1 month and in eight patients at 3-month follow-up (figure 3), although these changes were not statistically different from baseline.

\section{Renal function}

Renal disease was assessed by urinary protein excretion and GFR. Reduced $24 \mathrm{~h}$ proteinuria was seen in all patients after MSCT (figure 4) (see supplementary material). Post-MSCT proteinuria significantly decreased at 1 week (1430.7 \pm 306.3 vs $2538.0 \pm 382.3 \mathrm{mg}$ at baseline, $\mathrm{n}=12, \mathrm{p}<0.01)$, at 1 month (1385.8 \pm 273.6 vs $2540.8 \pm 340.1 \mathrm{mg}$ at baseline, $\mathrm{n}=13, \mathrm{p}<0.01$ ), at 3 months $(1273.8 \pm 194.0$ vs $2529.1 \pm 492.0 \mathrm{mg}$ at baseline, $\mathrm{n}=9, \mathrm{p}<0.05)$, at 6 months $(850.1 \pm 775.4$ vs $2655.2 \pm 1226.7 \mathrm{mg}$ at baseline, $\mathrm{n}=12, \mathrm{p}<0.01)$, at 12 months $(858.0 \pm 800.7$ vs $2505.0 \pm$ $1323.9 \mathrm{mg}$ at baseline, $\mathrm{n}=12, \mathrm{p}<0.01)$. GFR improved significantly in two patients with low GFR on study entry. In case 1, the GFR at 18 months was 47 and $46 \mathrm{ml} / \mathrm{min}$ in the left and right kidneys, respectively, compared to 33 and $32 \mathrm{ml} / \mathrm{min}$, respectively, before MSCT. In case 10, the GFR at 6 months was 53 and $45 \mathrm{ml} / \mathrm{min}$ the left and right kidneys, respectively, compared to 27 and $21 \mathrm{ml} / \mathrm{min}$, respectively before MSCT. Four subjects (cases 2, 9, 10 and 14) experienced significant improvement in their serum Cr levels: from 1.73 to 0.78 at 1 -month follow-up, from 5.36 to 2.41 at 3 months, from 4.40 to 2.08 at 1 month and from 2.55 to $0.90 \mathrm{mg} / \mathrm{dl}$ at 3 months, respectively.

\section{Non-LN-related manifestations}

Of 11 patients with a long history of fatigue and/or loss of weight and/or low grade fever, 8 became free from these manifestations based on patient reporting at 3 months after MSCT. All eight patients had a significant decrease in SLEDAI score compared with that of the baseline. Before transplantation, eight patients had skin rashes: four of them (cases 2, 4, 5 and 15), with severe cutaneous lesions healed completely at 1-month follow-up accompanied with decreased SLEDAI values, the other four (cases 3, 7, 8 and 12) responded at least partially and the lesions gradually faded at 3-month follow-up. One patient (case 10) had widespread skin ulcers due to herpes zoster. At 1 week after MSCT, the skin ulcers scabbed and the area involved reduced. Her lesions continued to heal 1 month after MSCT, paralleled with decreasing serum $\mathrm{Cr}$, blood urea nitrogen and proteinuria. Complete blood count at 1-month follow-up showed normal white cell count $\left(4.7 \times 10^{9}\right.$ cells/litre), haemoglobin $10.5 \mathrm{~g} / \mathrm{dl}$ and an improved platelet count $\left(95 \times 10^{9}\right.$ cells/litre).

Four out of six patients with involvement of the musculoskeletal system responded with decreased arthritis 1 month after MSCT. Serositis in two patients (ascites in cases 3 and 11) disappeared 1 week after MSCT. A total of 10 patients with refractory cytopoenia before enrolment all entered remission at 3-month follow-up. One patient with neurological system involvement (seizures and intractable headaches in case 2) had no seizures or headaches during follow-up after MSCT. Refractory hypertension in five patients was controlled satisfactorily after the transplantation accompanied with the amelioration of renal function abnormalities.

\section{Toxicities}

No serious adverse events were observed during or immediately after infusions of MSCs in any of the 15 patients with SLE. None of the patients developed graft versus host disease (GVHD) during follow-up. The most common adverse event noted was upper respiratory tract infections reported by some of the patients during follow-up visits. These mild infections were not thought to be related to the transplantation procedure. No serious infections occurred. None of the donors had any adverse events.

\section{Regulatory T cell measure}

To investigate the role of MSCT in Treg regulation, the percentages of CD4 Foxp3 $3^{+}$cells among peripheral blood mononuclear cells were detected by flow cytometric analysis (figure 5) (see supplementary material). The percentage of CD4 Foxp3 ${ }^{+}$at 1 week after MSCT increased to $4.58 \pm 0.51$ vs $2.56 \pm 0.37$ at baseline $(n=13, p<0.05)$. The percentages of Tregs cells at 3 and 6 months were $4.80 \pm 0.77(n=9)$ and $5.67 \pm 0.65(n=6)$, respectively, both significantly increased compared to the baseline level. CD4 Foxp3 ${ }^{+}$cells at 1 month were increased to $3.27 \pm 0.40$ vs $2.34 \pm 0.43$ at baseline $(n=9)$, but this change did not reach statistical significance $(p=0.19)$.

\section{DISCUSSION}

MSCs mediate immune responses in vitro and in vivo, in addition to being multipotential, easy to isolate and culture and possessing high expansion potential. Interest is growing in the role and therapeutic application of MSCs in GVHD and autoimmune diseases. Trials exploring MSCT in the treatment of steroidresistant, severe, acute GVHD achieved promising results. ${ }^{23}$ Our preclinical findings from experimental animals showed MSCT was effective in inhibiting disease progression in MLR-lpr mice. The $24-\mathrm{h}$ proteinuria, serum $\mathrm{Cr}$, anti-dsDNA antibodies and 


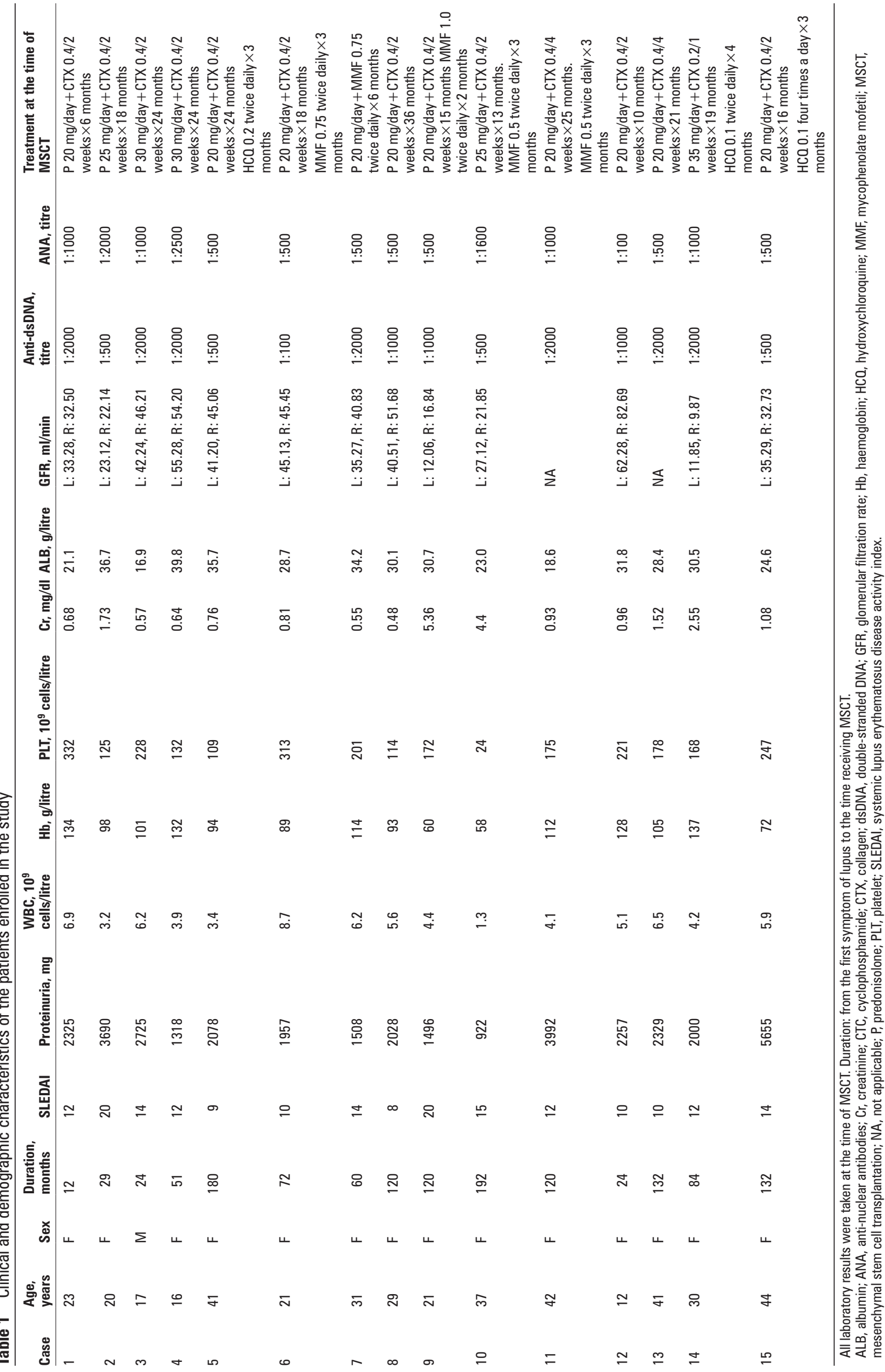


Table 2 Clinical symptoms and manifestations at the time of MSCT

\begin{tabular}{lc}
\hline Symptoms and manifestations & No. of patients \\
\hline Fatigue, loss of weight, low grade fever & 11 \\
Skin rash, Raynaud's phenomenon, alopecia & 8 \\
Arthritis, arthralgia, muscle pain, weakness & 6 \\
Serositis & 2 \\
Anaemia, leucocytopoenia, thrombocytopoenia & 10 \\
Lupus encephalopathy & 1 \\
Refractory hypertension & 5 \\
Proteinuria & 15 \\
\hline
\end{tabular}

MSCT, mesenchymal stem cell transplantation.

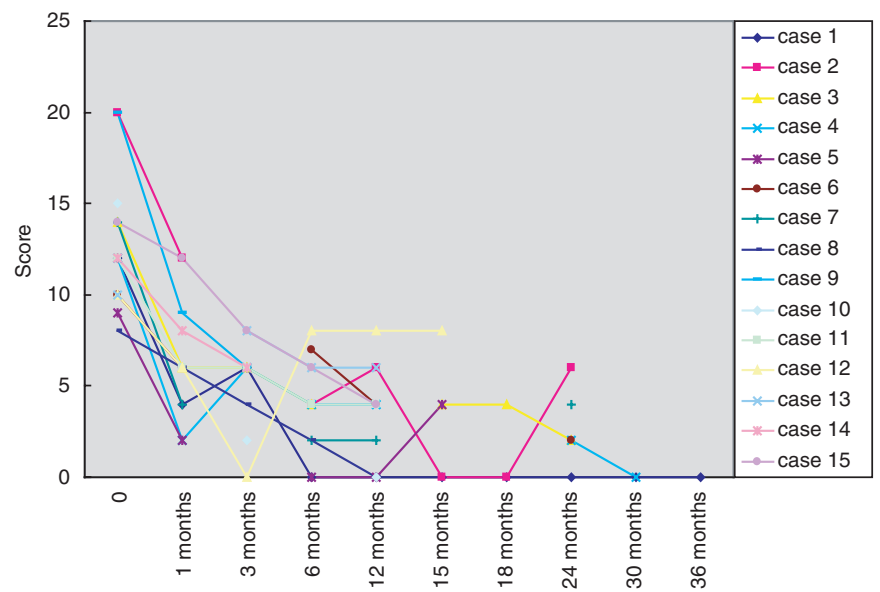

Figure 1 Systemic lupus erythematosus disease activity index (SLEDAI) scores in 15 patients with refractory systemic lupus erythematosus before and after mesenchymal stem cells transplantation. The SLEDAI score in all patients decreased after transplantation. The differences were significant on follow-up at 1, 3, 6, 12 and 24 months compared to the original indexes at the time of transplantation (all $p<0.05$, by paired $t$ test for groups). Score was 0 at the time of transplantation.

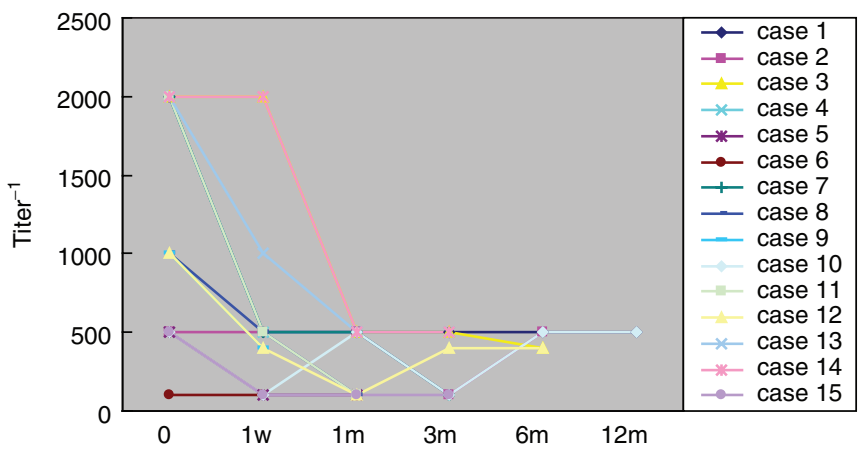

Figure 2 Anti-double-stranded DNA (dsDNA) antibody titres at the time of entry into the study and during the follow-up period in 15 patients with refractory systemic lupus erythematosus. Differences from baseline were significant at 1 and 3 months $(p<0.05)$.

Th2 subpopulations all decreased after MSCT. Data presented herein indicate that 15 patients with refractory SLE had reduced disease activity following treatment with MSCT. In these 15 patients, prednisolone was tapered stepwise successfully. At this time, a total of 13 patients were followed for more than 12 months, with the $24 \mathrm{~h}$ proteinuria excretion decreased significantly in 7 patients and normalised in 5 patients. Four patients showed remarkable improvement with SLEDAI scores of 0 at 12-month follow-up. Sustained clinical remission has lasted for

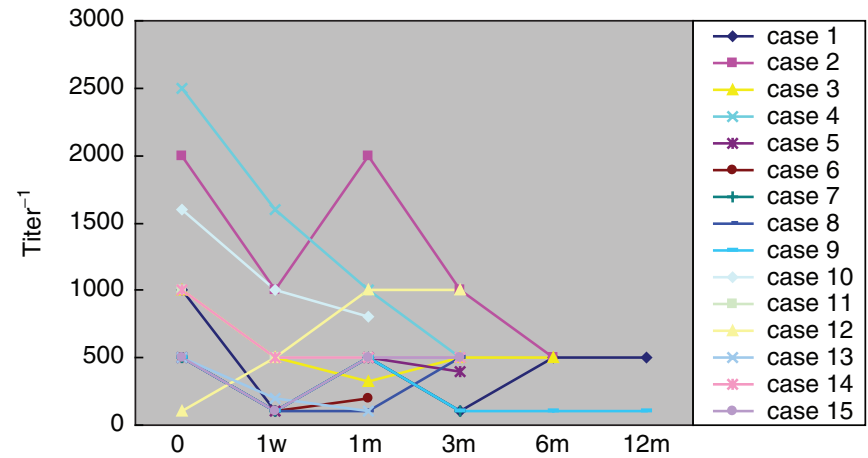

Figure 3 Anti-nuclear antibody (ANA) titres at the time of entry into the study and during the follow-up period in 15 patients with refractory systemic lupus erythematosus. Titres of ANA improved at 1-month and 3-month follow-up.

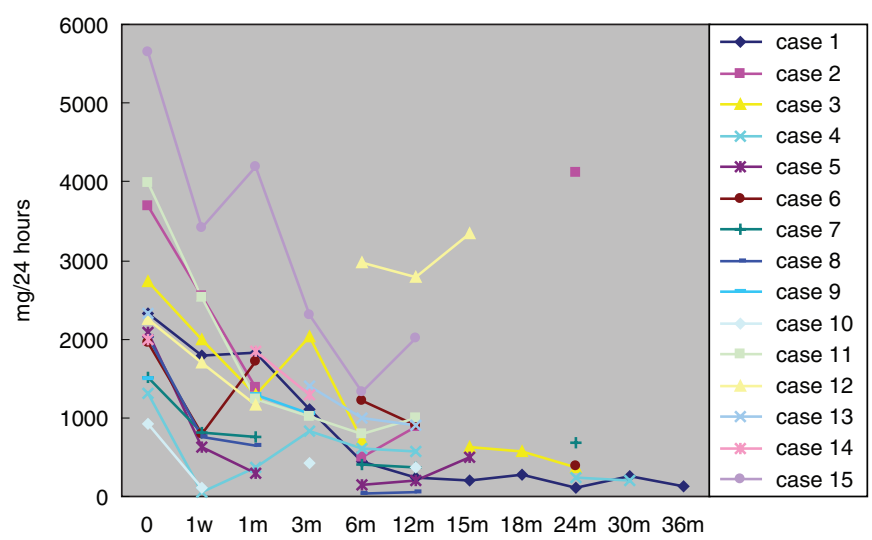

Figure 4 Results for 24-h proteinuria in 15 patients with refractory systemic lupus erythematosus before and after mesenchymal stem cells transplantation (MSCT). Reduced proteinuria was commonly seen during the follow-up period after MSCT at 1, 3, 6, 12 and 24 months (all $\mathrm{p}<0.05$ ).

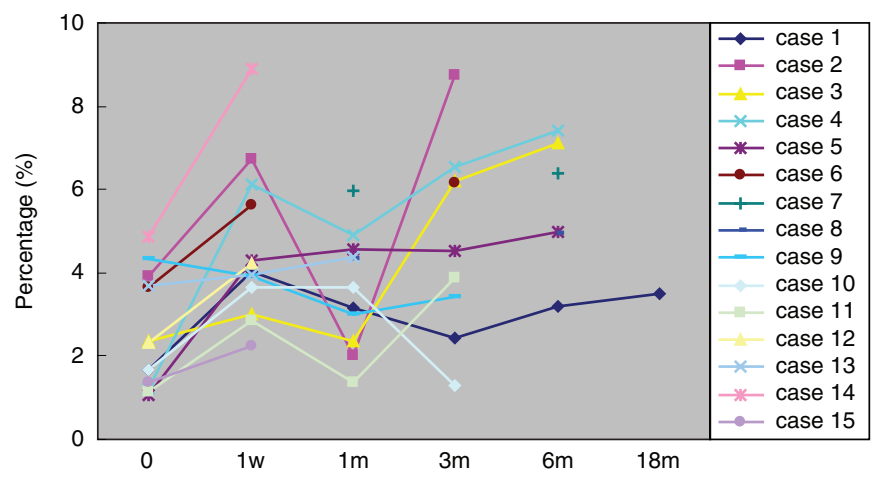

Figure 5 The percentage of regulatory T cells (Tregs) in 15 patients with refractory systemic lupus erythematosus before and after mesenchymal stem cells transplantation (MSCT). The percentage of Tregs signifi cantly increased at 1 week, 3 months and 6 months following MSCT $(p<0.05)$.

another 24 months in one of these four patients. A total of six patients were followed for more than 24 months, with the $24 \mathrm{~h}$ proteinuria excretion declined significantly in one patient and normalised in three patients. One patient had discontinued all the other immunosuppressants with a gradually taper of prednisone dosage after MSCT and had a SLEDAI score of 0 with prednisone $5 \mathrm{mg} /$ day at 12 -month follow-up. No patients had any serious adverse events either during or after infusion. In view 
of the lessened doses of prednisolone and immunosuppressants in all patients after the MSCT, we believe that it was the MSCT that was the causative factor in the potent therapeutic effect, not ongoing CYC or corticosteroids.

At present, little is known about mechanisms by which allogeneic MSCT might improve lupus. We hypothesise the disease modulation is induced by several mechanisms. First, MSCs work as immunomodulators by producing soluble factors induced upon stimulation. Imbalance of cytokine homeostasis is a prominent feature of experimental and human SLE. Many studies revealed increased levels of Th2 cytokines in murine lupus-prone strains as well as in patients with SLE. ${ }^{24-27}$ Soluble Th1 and Th2 cytokines were measured by ELISA in serum of patients with SLE prior to and after transplantation (data not shown). We found a trend towards increased levels of interferon $\gamma$ and tumour necrosis factor $\alpha$ suggesting a polarisation toward a Th1 phenotype in the Th1/Th2 balance after MSCT.

Second, MSCT may be associated with expansion of Tregs, which suppress the activity of autoreactive $T$ cells and play a pivotal role in the maintenance of self-tolerance. ${ }^{28}$ The pathogenesis of SLE may be associated with a defect in the homeostatic control of different Tregs subsets. ${ }^{29} 30$ The frequency and function of Tregs was significantly deficient in patients with active SLE. ${ }^{3132}$ At the same time, Foxp3 is described as the master controlling gene for the development and function of Tregs. ${ }^{33}$ To date, Foxp3 remains the best marker for analysis and quantification of Tregs population and has been reported to correlate with the function of Tregs. The mRNA level and protein expression of Foxp3 decreased in patients with new-onset active lupus. ${ }^{34}$ Accordingly, Foxp $3^{+}$CD4 Tregs were assessed in the peripheral blood of patients prior to and after transplantation. The percentage of Treg cells increased significantly at 1 week, 3 months and 6 months after MSCT. We speculate that the augmentation of Tregs may be one of the vital mechanisms for the therapeutic effect of MSCs.

The third presumed mechanism of effect in lupus is based on the multipotentiality of MSCs. Many studies showed that human BM-MSCs are capable of differentiating into endothelial cells in vitro. ${ }^{35}$ In addition, patients with SLE have increased levels of endothelial cell apoptosis by serum IgG. ${ }^{36}$ The pathological changes in the endothelial cells are believed to be one of the factors initiating nephron damage in LN. ${ }^{37} 38$ We hypothesise that, to some extent, differentiation of MSCs to endothelial cells reconstructs the structure of the nephron and improves renal function. Presently no data exist that confirm or refute this hypothesis.

In summary, this uncontrolled study showed efficacy of MSCT in 15 patients with refractory SLE, albeit with a short follow-up length. Randomised clinical studies are needed to compare MSCT with more conventional approaches. Once efficacy is confirmed, further clinical trials are needed to standardise study entry criteria, clinical response criteria, post-transplant immunosuppression schedule and treatment of relapses.

Acknowledgements This study was supported by grants from the National Natural Science Foundation of China (no. 30972736, 30772014); Jiangsu Province Science and Technology Achievement Transformation Foundation (BA2009124); Chinese National 115 Supporting Program (2008BAI59B02); Jiangsu Province Natural Science Foundation (BK2009034); Jiangsu Province 135 Talent Foundation (RC2007002); Jiangsu Province 'Six Summit Talent' Foundation and Nanjing Health Bureau Young Scientists Launching Project (OYK09174).

Funding This study was supported by grants from the National Natural Science Foundation of China (no. 30772014), National 115 Supporting Program (2008BAI59B02), Jiangsu Province 135 Talent Foundation (RC2007002), Jiangsu
Province Natural Science Foundation and Jiangsu Province Six Summit Talent Foundation.

Patient consent Obtained.

Ethics approval This study was conducted with the approval of the Ethics Committee at Drum Tower Hospital.

Provenance and peer review Not commissioned; externally peer reviewed.

Contributors $\mathrm{HZ}$ analysed data and approved the manuscript; JL analysed data, wrote the paper and approved the manuscript; $\mathrm{BH}$ and HW treated patients and approved the manuscript; LL, SS, YH, XZ, GSG and LS wrote the paper and approved the manuscript.

\section{REFERENCES}

1. Pepper R, Griffith M, Kirwan C, et al. Rituximab is an effective treatment for lupus nephritis and allows a reduction in maintenance steroids. Nephrol Dial Transplant 2009;24:3717-23.

2. Nannini C, Crowson CS, Matteson EL, et al. Mycophenolate mofetil is effective in reducing disease flares in systemic lupus erythematosus patients: a retrospective study. Lupus 2009;18:394-9.

3. Ramos-Casals M, Soto MJ, Cuadrado MJ, et al. Rituximab in systemic lupus erythematosus: a systematic review of off-label use in 188 cases. Lupus 2009;18:767-76.

4. Houssiau FA, Vasconcelos C, D'Cruz D, et al. The 10-year follow-up data of the euro-lupus nephritis trial comparing low-dose and high-dose intravenous cyclophosphamide. Ann Rheum Dis 2010;69:61-4.

5. Burt RK, Traynor A, Statkute L, et al. Nonmyeloablative hematopoietic stem cell transplantation for systemic lupus erythematosus. JAMA 2006;295:527-35.

6. Farge D, Labopin M, Tyndall A, et al. Autologous hematopoietic stem cell transplantation for autoimmune diseases: an observational study on 12 years' experience from the European group for blood and marrow transplantation working party on autoimmune diseases. Haematologica 2010;95:284-92.

7. Pittenger MF, Mackay AM, Beck SC, et al. Multilineage potential of adult human mesenchymal stem cells. Science 1999;284:143-7.

8. Jiang $\mathbf{Y}$, Jahagirdar BN, Reinhardt RL, et al. Pluripotency of mesenchymal stem cells derived from adult marrow. Nature 2002;418:41-9.

9. Pittenger MF, Martin BJ. Mesenchymal stem cells and their potential as cardiac therapeutics. Circ Res 2004;95:9-20.

10. Schwartz RE, Reyes M, Koodie L, et al. Multipotent adult progenitor cells from bone marrow differentiate into functional hepatocyte-like cells. J Clin Invest 2002;109:1291-302.

11. Satake K, Lou J, Lenke LG. Migration of mesenchymal stem cells through cerebrospinal fluid into injured spinal cord tissue. Spine 2004;29:1971-9.

12. Le Blanc K, Tammik L, Sundberg B, et al. Mesenchymal stem cells inhibit and stimulate mixed lymphocyte cultures and mitogenic responses independently of the major histocompatibility complex. Scand J Immunol 2003;57:11-20.

13. Klyushnenkova E, Mosca JD, Zernetkina V, et al. T cell responses to allogeneic human mesenchymal stem cells: immunogenicity, tolerance, and suppression. J Biomed Sci 2005;12:47-57.

14. Aggarwal S, Pittenger MF. Human mesenchymal stem cells modulate allogeneic immune cell responses. Blood 2005;105:1815-22.

15. Jiang $\mathbf{X X}$, Zhang $Y$, Liu B, et al. Human mesenchymal stem cells inhibit differentiation and function of monocyte-derived dendritic cells. Blood 2005;105:4120-6.

16. Sotiropoulou PA, Perez SA, Gritzapis AD, et al. Interactions between human mesenchymal stem cells and natural killer cells. Stem Cells 2006;24:74-85.

17. Ikehara $\mathbf{S}$. Bone marrow transplantation for autoimmune diseases. Acta Haematol 1998;99:116-32.

18. Sun LY, Zhang HY, Feng XB, et al. Abnormality of bone marrow-derived mesenchymal stem cells in patients with systemic lupus erythematosus. Lupus 2007;16:121-8.

19. Sun L, Akiyama $K$, Zhang H, et al. Mesenchymal stem cell transplantation reverses multiorgan dysfunction in systemic lupus erythematosus mice and humans. Stem Cells 2009;27:1421-32.

20. Nie Y, Lau CS, Lie AK, et al. Defective phenotype of mesenchymal stem cells in patients with systemic lupus erythematosus. Lupus 2010; (In Press)

21. Carrion F, Nova E, Ruiz C, et al. Autologous mesenchymal stem cell treatment increased $T$ regulatory cells with no effect on disease activity in two systemic lupus erythematosus patients. Lupus 2010;19:317-22.

22. Zhou K, Zhang $\mathrm{H}$, Jin $\mathrm{O}$ et al. Transplantation of human bone marrow mesenchymal stem cell ameliorates the autoimmune pathogenesis in MRL/lpr mice. Cell Mol Immunol 2008;5:417-24.

23. Le Blanc K, Rasmusson I, Sundberg B, et al. Treatment of severe acute graftversus-host disease with third party haploidentical mesenchymal stem cells. Lancet 2004;363:1439-41.

24. Richaud-Patin Y, Alcocer-Varela J, Llorente L. High levels of TH2 cytokine gene expression in systemic lupus erythematosus. Rev Invest Clin 1995;47:267-72.

25. Horwitz DA, Gray JD, Behrendsen SC, et al. Decreased production of interleukin-12 and other Th1-type cytokines in patients with recent-onset systemic lupus erythematosus. Arthritis Rheum 1998:41:838-44. 
26. Chen YC, Ye YL, Chiang BL. Establishment and characterization of cloned CD4- CD8alphabeta-T cell receptor (TCR)-bearing autoreactive T cells from autoimmune NZB $x$ NZW F1 mice. Clin Exp Immunol 1997;108:52-7.

27. Gröndal G, Gunnarsson I, Rönnelid J, et al. Cytokine production, serum levels and disease activity in systemic lupus erythematosus. Clin Exp Rheumatol 2000; 18:565-70.

28. Bennett CL, Christie J, Ramsdell F, et al. The immune dysregulation, polyendocrinopathy, enteropathy, $\mathrm{X}$-linked syndrome (IPEX) is caused by mutations of FOXP3. Nat Genet 2001;27:20-1.

29. Suen JL, Li HT, Jong YJ, et al. Altered homeostasis of CD4(+) FoxP3(+) regulatory T-cell subpopulations in systemic lupus erythematosus. Immunology 2009;127:196-205

30. Hahn BH, Anderson M, Le E, et al. Anti-DNA Ig peptides promote Treg cell activity in systemic lupus erythematosus patients. Arthritis Rheum 2008;58:2488-97.

31. Lyssuk EY, Torgashina AV, Soloviev SK, et al. Reduced number and function of CD4+CD25highFoxP3 + regulatory T cells in patients with systemic lupus erythematosus. Adv Exp Med Biol 2007;601:113-19.
32. Valencia X, Yarboro $C$, Illei G, et al. Deficient CD4+CD25high T regulatory cell function in patients with active systemic lupus erythematosus. J Immunol 2007;178:2579-88.

33. Fontenot JD, Rudensky AY. A well adapted regulatory contrivance: regulatory $T$ cell development and the forkhead family transcription factor Foxp3. Nat Immunol 2005:6:331-7.

34. Zhang B, Zhang X, Tang F, et al. Reduction of forkhead box P3 levels in CD4+CD25 high T cells in patients with new-onset systemic lupus erythematosus. Clin Exp Immunol 2008:153:182-7.

35. Oswald J, Boxberger S, Jørgensen B, et al. Mesenchymal stem cells can be differentiated into endothelial cells in vitro. Stem Cells 2004;22:377-84.

36. van Paassen P, Duijvestijn A, Debrus-Palmans $L$, et al. Induction of endothelial cell apoptosis by IgG antibodies from SLE patients with nephropathy: a potential role for anti-endothelial cell antibodies. Ann N Y Acad Sci 2007;1108:147-56.

37. Kinsey GR, Li L, Okusa MD. Inflammation in acute kidney injury. Nephron Exp Nephrol 2008;109:e102-7.

38. Morioka T. [Role of glomerular endothelial cells in glomerular disease]. Nippon Jinzo Gakkai Shi 2008;50:547-53. 